\title{
RESUSCITATION OF INFANTS BORN AT 22-26 WEEKS GESTATION WITH ROOM AIR
}

\author{
M. Ogur ${ }^{1}$, G. Sjörs ${ }^{1}$, G. Holmström ${ }^{2}$
}

\section{${ }^{I}$ Department of Neonatology, University Childrens' Hospital, ${ }^{2}$ Department of Ophthalmology, University} Hospital, Uppsala, Sweden

Background: Administration of extra oxygen $\left(\mathrm{O}_{2}\right)$ during resuscitation of infants born extremely prematurely has been clinical practice. However, this may cause excessive oxidative stress. Since 2007, the policy at the University Hospital has been to initiate resuscitation with room air at all gestational ages.

Objective: To investigate the feasibility of resuscitating extremely premature infants with room air.

Methods: Inborn infants, born at 22-26 weeks of gestation in 2007-2009 ( $\mathrm{n}=99$ ), were included in a retrospective analysis. Data was extracted from the Swedish neonatal quality register, supplemented with information from patient's records. Infants were grouped according to oxygen supplementation during initial stabilisation: The Room Air Group $(\mathrm{n}=43)$ received no additional $\mathrm{O}_{2}$; the Intermediate Group $(\mathrm{n}=18)$ received $\leq 40 \% \mathrm{O}_{2}$ for $<1$ hour; and the Oxygen Group $(\mathrm{n}=38)$ received $>40 \% \mathrm{O}_{2}$. Infants born at $22-26$ weeks gestation in 2004-2006, when supplemental $\mathrm{O}_{2}$ was routinely administered during resuscitation, were used as control group $(\mathrm{n}=100)$. There were no significant differences in background variables between study groups.

Results:

\begin{tabular}{|l|l|l|l|l|l||}
\hline \multicolumn{1}{|c|}{ Outcome } & Oxygen Group & $\begin{array}{c}\text { Intermediate } \\
\text { Group }\end{array}$ & $\begin{array}{c}\text { Room Air } \\
\text { Group }\end{array}$ & Control Group & \\
\hline Mortality & $11 / 38(29,0 \%)$ & $2 / 18(11,1 \%)$ & $8 / 43(18,6 \%)$ & $25 / 100(25,0 \%)$ & NS \\
\hline IVH $\geq$ grade 3 & $5 / 38(13,2 \%)$ & $2 / 18(11,1 \%)$ & $4 / 43(9,3 \%)$ & $10 / 100(10,0 \%)$ & NS \\
\hline NEC & $2 / 38(5,3 \%)$ & $1 / 18(5,6 \%)$ & $4 / 43(9,3 \%)$ & $4 / 100(4,0 \%)$ & NS \\
\hline ROP $\geq$ grade 3 & $8 / 29(27,6 \%)$ & $3 / 16(18,8 \%)$ & $12 / 35(34,3 \%)$ & $20 / 80(25,0 \%)$ & NS \\
\hline Severe BPD & $9 / 2(31,0 \%)$ & $2 / 16(12,5 \%)$ & $4 / 35(11,4 \%)$ & $21 / 80(26,3 \%)$ & NS \\
\hline
\end{tabular}

[There were no significant differences in outcomes.]

Conclusions: The results indicate that resuscitation and initial stabilisation of extremely premature infants with room air is feasible, and does not increase mortality or major morbidity. 